\title{
Retraction: 802.11B Considered Harmful
}

\author{
Zhong Chen \\ Department of Mathematics and Computer Science, \\ Fuqing Branch of Fujian Normal University, \\ Fuqing 350300, China \\ czcat@163.com
}

Several conference proceedings have been infiltrated by fake submissions generated by the SCIgen computer program. Due to the fictional content the chapter "802.11B Considered Harmful" by "Zhong Chen" has been retracted by the publisher. Measures are being taken to avoid similar breaches in the future. 